\title{
Effects and mechanisms of a mindfulness-based intervention on insomnia
}

\section{Hye-Geum Kim}

Department of Psychiatry, Yeungnam University College of Medicine, Daegu, Korea

Received: December 8, 2020

Accepted: December 16, 2020

Corresponding author:

Hye-Geum Kim, MD, PhD

Department of Psychiatry,

Yeungnam University College of

Medicine, 170 Hyeonchung-ro,

Nam-gu, Daegu 42415, Korea

Tel: +82-53-620-3340

Fax: +82-53-657-3921

E-mail: psykhg@yu.ac.kr

\begin{abstract}
Medication alone is not sufficient to treat insomnia. In addition, the side effects of sleep medications themselves cannot be ignored during treatment. Insomnia begins with poor sleep quality and discomfort, but as it continues, patients fall into a vicious circle of insomnia with negative thoughts and dysfunctional and distorted perceptions related to sleep. Mindfulness-based intervention for insomnia corrects these sequential cognitive and behavioral processes. The mindfulness technique basically recognizes all the thoughts, feelings, and experiences that occur to us as they are, nonjudgmentally, and then trains them to return to the senses of our body. In this way, while noticing all the processes of the sequential vicious cycle and training them to return to our bodies (e.g., breathing), mindfulness determines whether we are really sleepy or just fatigued. This mindfulness-based intervention can be a useful nonpharmaceutical intervention for insomnia, and its stability and efficacy has been proven by many studies.
\end{abstract}

Keywords: Insomnia; Meditation; Mindfulness; Sleep; Sleep wake disorder

\section{Introduction}

Insomnia is often treated with medication. While such treatments provide only a temporary improvement in sleep disorders for some people, the benefits after drug discontinuation are often diminished, with the negative effects on daytime functioning and risk of addiction [1-3]. One of the common nonpharmacological interventions for insomnia is poor sleep hygiene education, which targets changes in daily behavior and environmental factors that cause sleep deprivation. Among standard clinical treatments, cognitive behavioral therapy (CBT) focuses on regulating sleep needs and modifying sleep expectations, attitudes, and beliefs $[1,4]$. Since the report of a randomized controlled trial (RCT) published in the Journal of the American Medical Association (JAMA) in 2015 revealed the effects of mindfulness-based interventions (MBIs) on insomnia, a nonpharmacological approach has been expected for mindfulness-based treatments for insomnia [5].
Mindfulness and mindfulness meditation are ways to recognize all thoughts, feelings, and body sensations that occur to us as they are, observe them without judgment, and accept them as they are. By observing negative thoughts and feelings in a nonjudgmental way, we do not automatically respond to such thoughts and feelings, and ultimately do not identify them with us and look at them as a kind of mental event that is not absolutely true and can come to us for a while and then disappear [6]. It appears from published findings that there is no longer any doubt that these MBI have therapeutic effects in mental health medicine. A recently published randomized controlled study on sleep disorders [7], randomized controlled study of patients with depression $[8,9]$, anxiety disorders [10], psychosomatic disorders including pain [11], substance abuse disorders [12], and eating disorders [13], all showed significant effects compared to the control group. In addition to the effects of MBIs on each disease, studies are continuing to elucidate the underlying mechanisms of MBI [14]. Moreover, attempts have

Copyright (C) 2021 Yeungnam University College of Medicine

This is an Open Access article distributed under the terms of the Creative Commons Attribution Non-Commercial License (http://creativecommons.org/licenses/by-nc/4.0/) which permits unrestricted non-commercial use, distribution, and reproduction in any medium, provided the original work is properly cited. 
been made to reveal the mechanism of the effect of $\mathrm{MBI}$ in a neurobiological way and to investigate this using neuroimaging techniques $[15,16]$. In recent years, there have been reports that MBI affects the activity of telomerase as well as the improvement of immunological function $[7,17,18]$.

This paper reviewed the effect of MBI on insomnia and also evaluated the mechanistic evidence to aid in future clinical application.

\section{Vicious cycle of insomnia}

Patients with chronic insomnia describe their condition as a "vicious cycle." In other words, insomnia worsens as the effort and desire to sleep increases. A strong desire to get more sleep and avoid daytime fatigue leads to a state of feeling trapped between the states of drowsiness and awakening [19]. Several models have been proposed to explain the pathophysiology of insomnia. Among them, Shallcross and Visvanathan [18] presented a model of insomnia that integrated core cognitive and behavioral processes from several extant theoretical frameworks. Insomnia is generally initiated and persisted by the following continuous cognitive and behavioral processes: (1) excessive daytime and nighttime rumination (i.e., excessive daytime and nighttime anxiety and rumination of sleep); (2) primary arousal (i.e., physiological arousal occurs with the first negative assessment of daytime problems caused by sleep deprivation); (3) secondary arousal (i.e., negative secondary or metacognitive judgment of initial arousal and subsequent continuation of physiological arousal); (4) excessive monitoring and selective attention to internal and/or external sleep cues that are consistent or inconsistent with sleep onset; (5) dysfunctional perceived need for control and engagement in sleep effort (i.e., actively taking a nap or increasing sleep opportunities); and (6) distorted perceptions about sleep impairment (i.e., perceptions about sleep impairment as excessively serious). Practical examples for each of these sequential cognitive and behavioral processes would be the following. (1) Rumination: "All day long, there are useless thoughts of sleep in my head." (2) Primary arousal: "What if I have a problem with my work tomorrow?" (3) Secondary arousal: "Why am I doing this? I feel bad and irritable." (4) Selective attention/sleep monitoring: "How did I fall asleep normally? I keep observing the feeling of falling asleep." (5) Dysfunctional perceived need for sleep: "I have to take a nap tomorrow, otherwise I can't work." (6) Distorted perceptions: "I'm desperate. If my sleep time is short, I am going to get tired and ruin my work all day tomorrow. What if I get fired?" Misunderstandings of sleep deprivation often lead to excessive negative perceptions of sleep, thus strengthening the vicious cycle of chronic insomnia.
CBT for insomnia (CBT-I) is one of the most widely studied nonpharmacological therapies for insomnia [20]. CBT-I has evolved as a multi-component therapeutic approach, combining the following: (1) cognitive strategies, such as thought restructuring to change sleep-related dysfunctional beliefs and attitudes; (2) behavioral techniques, such as sleep restriction and stimulus control, to promote healthy sleep habits. While this is a good way to mediate the pathophysiological mechanisms of insomnia, a significant proportion (19\%-26\%) of individuals do not benefit from CBT-I, and the average overall improvement among those who do respond is only $50 \%$ to $60 \%$ [21]. In order to resolve the ironic situation in which the more obsessed with falling asleep, the more sleep will run away, something other than a cognitive approach is necessary.

\section{Theoretical rationale of mindfulness- based interventions for insomnia}

While the effectiveness of MBI has been proven by many studies, consistent results have not been reported on the mechanism of therapy. In a review article published in 2016 [14], the psychological mechanisms suggested by existing researchers were systematically reviewed and published; however, each researcher had prioritized these differently. In addition, attempts are being made to clarify the mechanism of the effect of MBI neurobiologically and to investigate this by using neuroimaging techniques $[15,16]$.

During mindfulness meditation, there is a physiological change in the "wakeful hypometabolic state" [22]. The activity of the sympathetic nervous system decreases and the activity of the parasympathetic nervous system, which is important for relaxation and rest, increases, but this state is known to be one that is clearly different from the state of simply resting or sleeping qualitatively and quantitatively [23]. In other words, in addition to relaxing the body, it aims to reduce mental activity by relaxing the mind, thereby providing feedback to the body, leading to deeper physical relaxation. This has been proven in studies that measure physiological changes in the body while practicing mindfulness meditation $[22,23]$. Body relaxation, such as simple muscle relaxation, is associated with the activation of primary and secondary motor regions. The activity of the paralimbic brain regions, which mediate sympathetic nerve stimulation, such as the anterior cingulate and insula, is known to be a change in both relaxation and mindfulness meditation. It is known that certain areas other than these are additionally activated, and many experienced meditators have reported activation of frontolimbic and frontoparietal neural networks [2429]. More interesting to note, is that larger, intensified activation is observed in the frontoparietal attention networks in experienced 
meditators than in novice meditators with little experience. If mindfulness meditation is not different from simple general relaxation, short-term and long-term meditators are expected to relax similarly and not differ in their physiological or neurophysiological characteristics. These physical and mental relaxation states are helpful for mental as well as physical arousal that occurs in the vicious cycle of insomnia.

The psychological mechanisms by which MBI influences insomnia are as follows. First, MBI helps in insomnia by improving metacognitive awareness. One of the important elements of mindfulness is based on the fact that "there is nothing eternal and unchanging" which is central to Buddhist psychology. With mindfulness meditation training, all experiences are observed as they occur and disappear. In the process, we become aware of constantly changing experiences, and thus, we understand that all experiences are temporary [30]. This process has been called reperceiving or decentering [31] and has been described as the development of an observer perspective [32]. In the literature dealing with MBI, metacognitive awareness, a variety of terms refer to the ability to observe one's own thoughts or feelings as transient events of the mind, rather than being true and accurate in themselves. It was somewhat mixed with decentering, defusion, distancing, and reperceiving [33]. Through the mindfulness training process, it becomes possible to see the content of consciousness, that is, the moment-to-moment experience more clearly and objectively without identifying oneself with one's thoughts. Thus, it becomes a therapeutic process that introduces a 'space' between one's own 'perception' and 'reaction' by leaving the individual's immediate experience from the observer's point of view for insight and analysis of habitual patterns of emotions and behaviors. Indeed, evidence that mindfulness meditation training influences metacognitive awareness has been reported in several studies [34,35]. Metacognitive awareness helps people with insomnia to become aware of their thoughts and experiences as and when they are trapped in a vicious cycle of insomnia, which breaks the link to the next step. In fact, there are reports that MBI reduces rumination, which is common in patients with insomnia through metacognitive awareness [36].

Attention control is also a major psychological mechanism by which MBI affects insomnia. The development of attention is one of the key elements of mindfulness meditation training. A general guideline for meditation in line with the mindfulness meditation tradition is "Focus on your breathing in and out. Keep your attention without distracting. If you get distracted, quietly turn your attention to your breath and start over [37]." Attention training improves the ability to maintain nonjudgmental awareness of thought patterns, assumptions, and sensory perception. This awareness helps keep your thoughts and emotions away from being overly intense. With mindfulness meditation, we train to sustain attention to your breathing and redirect your attention to our breathing as an anchor whenever our thoughts wander. This attention control is helpful in rumination, arousal, and selective attention/sleep monitoring processes in the vicious cycle of insomnia.

The next psychological mechanism of MBI is the reduction of automatic thoughts and self-referential thinking. Automatic thinking begins unconsciously and is not easy to interfere with or prevent. In other words, when consciousness cannot consciously attract attention, the default mode network (DMN) starts involuntarily [38]. Objective perception of thinking through mindfulness meditation interprets thinking as 'just thoughts' and prevents unreasonable negative thinking from seeing as facts. Objective recognition of these automatic thoughts through mindfulness meditation is known as a major mechanism by which MBI reduces depression, anxiety, and stress [39]. Parts of the DMN, especially cortical midline structures (CMS), include the medial prefrontal cortex, the anterior cingulate cortex, and the posterior medial cortices, which are automatic thinking $[40,41]$. It is known to play an important role in CMS, and it has been reported that decreased activity of CMS is associated with a decrease in automatic thinking. CMS is also involved in self-referential thinking, which is a type of automatic thinking known to be associated with mood and anxiety disorders. MBI weakens nonadaptive habitual self-view by affecting the DMN area, especially CMS [16]. Reducing these negative and automatic thoughts through MBI can reduce negative, but extremely subjective, self-referencing thoughts that occur during insomnia. This helps to reduce primary and secondary arousal in the vicious cycle of insomnia.

In addition, meditation is a training of acceptance. Acceptance is a nonjudgmental process, acknowledging and accepting the experience of the mind and body at the present moment as it is. The automatic reaction of the mind hates all pain and runs blindly. However, this autopilot reaction is more painful. In a way of life that accepts pain and can coexist, acknowledging reality and not escaping, it becomes active approval, not passive defeat. One way to accept pain is through self-compassion. The concept of self-compassion is related to mindfulness [42]. Self-compassion, defined by Neff [41], consists of three components. The first is self-kindness, which refers to an attitude that allows us to have a kind and understanding attitude to ourselves rather than being harsh and critical when we feel painful or inappropriate. The second is common humanity, which is to perceive one's own experience as part of a greater human experience than to see it as separating and isolating. The third is mindfulness, which refers to the ability to experience one's painful emotions and thoughts with a balanced awareness rather 
than overidentifying with them. As can be seen from the concept itself, mindfulness and self-compassion are very related, and one study reported the correlation between the total score on the mindfulness scale and the total score on the self-compassion scale [43]. In addition, studies have reported that changes in mindfulness can predict changes in self-compassion, and it has been argued that self-compassion partially mediates the relationship between mindfulness and well-being [44]. This acceptance relieves the pain of secondary arousal and continuous distorted and biased perceptions and thoughts that occur in insomnia. Increased acceptance of difficult thoughts, emotions, and physical sensations allowed them to let go of their desire (and desperate behaviors) to make sleep happen.

\section{Evidence of mindfulness-based interventions for insomnia}

Many studies have been conducted to verify the effect of MBI on insomnia. Representatively, the RCT published in the 2015 JAMA was randomized into two groups for those with moderate sleep disorder (Pittsburgh Sleep Quality Index [PSQI] > 5) among adult subjects (mean \pm standard deviation of age, $66.3 \pm 7.4$ years). A total of 49 patients were randomly assigned to the MBI or general sleep hygiene training for 6 weeks ( 2 hours per week), and participants in the MBI group showed significant improvement in PSQI compared to those in the control group. The mean difference between groups was 1.8 (95\% confidence interval, 0.6-2.9) and the effect size was 0.89 . MBI showed significant improvement in secondary health outcomes of insomnia symptoms, depressive symptoms, fatigue disorder, and fatigue intensity compared to the control group (all $p<0.05)$. Nuclear factor kappa B (NF- $\kappa \mathrm{B})$ concentration decreased significantly with time in both groups $(p<0.05)$. No differences were observed between the groups for anxiety, stress, and NF- $\kappa B$ [5]. Studies that include more objective indicators as variables have also been reported accordingly. In one study, mindfulness-based stress reduction (MBSR) and drug treatment groups were randomly assigned, and the Insomnia Severity Index (ISI), PSQI, sleep diaries, and wrist actigraphy were used as objective indicators. When comparing the results after 8 weeks with the baseline, sleep onset latency in the MBSR group decreased by 8.9 minutes $(p<0.05)$ [45]. In a 2015 study of breast cancer survivors, subjects were randomly assigned to the MBSR group and the usual care (UC) group, and objective variables, including actigraphy, were measured. As a result, after 12 weeks of objective sleep variable, MBSR group had a more significant effect than control group in the following areas; sleep efficiency $(78.2 \%$ of MBSR group vs. $74.6 \%$ of UC group, $p=0.04$ ), percent of sleep time ( $81.0 \%$ of MBSR group vs. $77.4 \%$ of UC group, $p=0.02$ ), and less number of waking bouts (93.5 in MBSR group vs. 118.6 in the UC group, $p<0.01$ ) [46].

In a 2014 study, a randomized, partially blinded, and noninferiority trial was conducted to determine whether MBSR was inferior to CBT-I for insomnia. This study was conducted in patients with insomniac cancer, and the evaluation was performed at baseline, immediately after the program, and after 3 months of follow-up. MBSR was inferior to CBT-I immediately after the program but showed noninferiority after follow-up $(p=0.02)$. Although CBT-I is associated with rapid and durable improvement and remains the best option for nonpharmacological treatment of insomnia, MBSR has brought about clinically significant changes in sleep and psychological outcomes [47]. In another three-arm and single-site RCT, 54 subjects were placed in the MBSR group, mindfulness-based therapy for insomnia (MBTI) group, or an 8-week selfmonitoring condition. Total wake time (TWT), Pre-Sleep Arousal Scale (PSAS), ISI, and objective sleep measurements were measured using laboratory polysomnography and wrist actigraphy. Subjects who received MBI (MBSR or MBTI) were found to be superior to the self-monitoring control group from baseline to postintervention in TWT (43.75 vs. 1.09), PSAS (7.13 vs. 0.16 ), and ISI (4.56 vs. 0.06 ). There was no significant difference between the MBSR and MBTI. From baseline to 6-month follow-up, MBTI showed a greater reduction in ISI score than $\operatorname{MBSR}(p<0.05)$, with the largest difference at the 3-month follow-up. Remission and response rates of MBTI and MBSR were maintained until 6 months follow-up, and MBTI showed the highest ratio of treatment remission (50\%) and response (78.6\%) at 6 months follow-up [48].

As these studies have increased recently, systematic reviews and meta-analysis results of these studies are also reported in literature. In the meta-analysis results of MBI insomnia published in 2020, participants in the MBI group showed a significant improvement in insomnia as measured by the PSQI $(p<0.00001)$ as compared to the control group. In this comprehensive meta-analysis, MBI appears to be effective in treating insomnia. However, the authors suggested that further research is needed to investigate the longterm effects of MBI on insomnia [49].

Each study has used somewhat different techniques for MBI as a basis for insomnia. The MBI that has accumulated the most evidence so far is MBSR, and in the case of mindfulness-based cognitive therapy, subjective improvement is reported, but the most objective evidence is insufficient [50,51]. In the case of MBTI, which is a more specific technique for insomnia, recent studies are underway, and several significant results described above have been reported accordingly. 


\section{Mindfulness-based approach to treatment of insomnia}

The mindfulness-based approach to insomnia incorporates key elements of MBI. In order to quantitatively report the effect on insomnia, conventional MBIs such as MBSR have been widely used as research results. In recent years, evidence for MBTI specialized in insomnia has also been collected accordingly. If the core elements of mindfulness are properly included, basically, any form of intervention will help with insomnia. However, the therapists will need to be embodied in the MBI; they should have medical knowledge, including that of sleep physiology.

The goal of MBTI is to increase awareness of mental and physical conditions that cause chronic insomnia and to develop adaptive ways for these undesirable conditions [52]. The practice of meditation, discussion, and daily monitoring of sleep and waking activity helps to enhance this awareness. Particular attention is paid to the mental and physical conditions of sleepiness and fatigue, and participants are taught to distinguish between these two conditions. Using awareness as a platform, you are trained to respond to sleep disorders with mindfulness techniques instead of automatically reacting by increasing effort to rest. Participants should avoid meditating to sleep at night. Instead, the meditations are used as a practice of cultivating awareness and mindfulness principles, not to be used as a relaxation strategy for falling asleep. It includes effectively managing emotional responses to sleep disturbance and daytime fatigue as well as reducing unwanted waking at night. As the program progresses, participants are taught to use mindfulness principles and behavioral strategies to work in these undesired conditions. Specific behavioral changes (sleep restriction and stimulus control) are empirically supported techniques for insomnia that complement the mindfulness principle and should be treated together. MBTI consists of three major components: (1) start with activities in the form of formal mindfulness meditation, including one quiet meditation and one movement meditation; (2) discussions are guided by MBTI leaders when participants are asked about their findings during meditation and their application to insomnia; and (3) the didactic period includes education about sleep and wake physiology and instructions for stimulus control and sleep restriction [52].

\section{Conclusion}

Insomnia begins with the discomfort of sleep itself, but as it continues, patients fall into a vicious circle with negative thoughts related to sleep. As such, those who experience chronic insomnia fall into a vicious cycle of "increasing sleep worsens as the desire and effort to sleep increases" which in turn leads to a strong desire to get more sleep and avoid daytime fatigue. MBI corrects sequential cognitive and behavioral processes and can be a useful nonpharmaceutical intervention for insomnia. In many studies, MBI for insomnia has been proven with stability and efficacy.

\section{Notes}

\section{Conflicts of interest}

No potential conflict of interest relevant to this article was reported.

\section{ORCID}

Hye-Geum Kim, https://orcid.org/0000-0002-9677-7011

\section{References}

1. Silber MH. Clinical practice: chronic insomnia. N Engl J Med 2005;353:803-10.

2. Nowell PD, Mazumdar S, Buysse DJ, Dew MA, Reynolds CF 3rd, Kupfer DJ. Benzodiazepines and zolpidem for chronic insomnia: a meta-analysis of treatment efficacy.JAMA 1997;278: 2170-7.

3. Morin CM, Colecchi C, Stone J, Sood R, Brink D. Behavioral and pharmacological therapies for late-life insomnia: a randomized controlled trial. JAMA 1999;281:991-9.

4. Montgomery P, Dennis J. Cognitive behavioural interventions for sleep problems in adults aged 60+. Cochrane Database Syst Rev 2003;(1):CD003161.

5. Black DS, O'Reilly GA, Olmstead R, Breen EC, Irwin MR. Mindfulness meditation and improvement in sleep quality and daytime impairment among older adults with sleep disturbances: a randomized clinical trial. JAMA Intern Med 2015;175: 494-501.

6. Korean NeuroPsychiatric Association. Textbook of neuropsychiatry. 3th ed. Seoul: IAmIsCompany; 2017. p. 365.

7. Falsafi N. A randomized controlled trial of mindfulness versus yoga: effects on depression and/or anxiety in college students. J Am Psychiatr Nurses Assoc 2016;22:483-97.

8. Michalak J, Probst T, Heidenreich T, Bissantz N, Schramm E. Mindfulness-based cognitive therapy and a group version of the cognitive behavioral analysis system of psychotherapy for chronic depression: follow-up data of a randomized controlled trial and the moderating role of childhood adversities. Psychother Psychosom 2016;85:378-80.

9. Hoge EA, Bui E, Marques L, Metcalf CA, Morris LK, Robinaugh DJ, et al. Randomized controlled trial of mindfulness 
meditation for generalized anxiety disorder: effects on anxiety and stress reactivity.J Clin Psychiatry 2013;74:786-92.

10. Feuille M, Pargament K. Pain, mindfulness, and spirituality: a randomized controlled trial comparing effects of mindfulness and relaxation on pain-related outcomes in migraineurs. J Health Psychol 2015;20:1090-106.

11. Shorey RC, Elmquist J, Gawrysiak MJ, Strauss C, Haynes E, Anderson $S$, et al. A randomized controlled trial of a mindfulness and acceptance group therapy for residential substance use patients. Subst Use Misuse 2017;52:1400-10.

12. Ruffault A, Carette C, Lurbe I Puerto K, Juge N, Beauchet A, Benoliel JJ, et al. Randomized controlled trial of a 12-month computerized mindfulness-based intervention for obese patients with binge eating disorder: the MindOb study protocol. Contemp Clin Trials 2016;49:126-33.

13. Gu J, Strauss C, Bond R, Cavanagh K. How do mindfulness-based cognitive therapy and mindfulness-based stress reduction improve mental health and wellbeing?: a systematic review and meta-analysis of mediation studies. Clin Psychol Rev 2015;37:1-12.

14. Tang YY, Hölzel BK, Posner MI. The neuroscience of mindfulness meditation. Nat Rev Neurosci 2015;16:213-25.

15. Marchand WR. Neural mechanisms of mindfulness and meditation: evidence from neuroimaging studies. World J Radiol 2014;6:471-9.

16. Alda M, Puebla-Guedea M, Rodero B, Demarzo M, Montero-Marin J, Roca M, et al. Zen meditation, length of telomeres, and the role of experiential avoidance and compassion. Mindfulness (NY) 2016;7:651-9.

17. Epel E, Daubenmier J, Moskowitz JT, Folkman S, Blackburn E. Can meditation slow rate of cellular aging?: cognitive stress, mindfulness, and telomeres. Ann N Y Acad Sci 2009; 1172:3453.

18. Shallcross AJ, Visvanathan PD. Mindfulness-based cognitive therapy for insomnia. In: Eisendrath SJ, editor. Mindfulness-based cognitive therapy. Springer International Publishing AG; 2016. p. 19-29.

19. Morin CM. Cognitive-behavioral therapy of insomnia. Sleep Med Clin 2006;1:375-86.

20. Taylor DJ, Pruiksma KE. Cognitive and behavioural therapy for insomnia (CBT-I) in psychiatric populations: a systematic review. Int Rev Psychiatry 2014;26:205-13.

21. Cahn BR, Polich J. Meditation states and traits: EEG, ERP, and neuroimaging studies. Psychol Bull 2006;132:180-211.

22. Jevning R, Wallace RK, Beidebach M. The physiology of meditation: a review: a wakeful hypometabolic integrated response. Neurosci Biobehav Rev 1992;16:415-24.
23. Brefczynski-Lewis JA, Lutz A, Schaefer HS, Levinson DB, Davidson RJ. Neural correlates of attentional expertise in longterm meditation practitioners. Proc Natl Acad Sci U S A 2007; 104:11483-8.

24. Lazar SW, Bush G, Gollub RL, Fricchione GL, Khalsa G, Benson $\mathrm{H}$. Functional brain mapping of the relaxation response and meditation. Neuroreport 2000;11:1581-5.

25. Hölzel BK, Ott U, Hempel H, Hackl A, Wolf K, Stark R, et al. Differential engagement of anterior cingulate and adjacent medial frontal cortex in adept meditators and non-meditators. Neurosci Lett 2007;421:16-21.

26. Farb NA, Segal ZV, Mayberg H, Bean J, McKeon D, Fatima Z, et al. Attending to the present: mindfulness meditation reveals distinct neural modes of self-reference. Soc Cogn Affect Neurosci 2007;2:313-22.

27. Newberg A, Alavi A, Baime M, Pourdehnad M, Santanna J, d'Aquili E. The measurement of regional cerebral blood flow during the complex cognitive task of meditation: a preliminary SPECT study. Psychiatry Res 2001;106:113-22.

28. Aftanas LI, Golocheikine SA. Human anterior and frontal midline theta and lower alpha reflect emotionally positive state and internalized attention: high-resolution EEG investigation of meditation. Neurosci Lett 2001;310:57-60.

29. Olendzki A. Unlimiting mind: the radically experiential psychology of Buddhism. Somerville (MA): Wisdom Publications; 2010.

30. Carmody J, Baer RA, Lykins EL, Olendzki N. An empirical study of the mechanisms of mindfulness in a mindfulness-based stress reduction program. J Clin Psychol 2009;65:613-26.

31. Kerr CE, Josyula K, Littenberg R. Developing an observing attitude: an analysis of meditation diaries in an MBSR clinical trial. Clin Psychol Psychother 2011;18:80-93.

32. Fresco DM, Moore MT, van Dulmen MH, Segal ZV, Ma SH, Teasdale JD, et al. Initial psychometric properties of the experiences questionnaire: validation of a self-report measure of decentering. Behav Ther 2007;38:234-46.

33. Teasdale JD, Moore RG, Hayhurst H, Pope M, Williams S, Segal $\mathrm{ZV}$. Metacognitive awareness and prevention of relapse in depression: empirical evidence. J Consult Clin Psychol 2002;70: 275-87.

34. Hargus E, Crane C, Barnhofer T, Williams JM. Effects of mindfulness on meta-awareness and specificity of describing prodromal symptoms in suicidal depression. Emotion 2010;10:34-42.

35. Teasdale JD. Metacognition, mindfulness and the modification of mood disorders. Clin Psychol Psychother 1999;6:146-55.

36. Smith H, Novak P. Buddhism: a concise introduction. New York: HarperCollins; 2003. 
37. Buckner RL, Andrews-Hanna JR, Schacter DL. The brain's default network: anatomy, function, and relevance to disease. Ann NY Acad Sci 2008;1124:1-38.

38. Marchand WR. Mindfulness-based stress reduction, mindfulness-based cognitive therapy, and Zen meditation for depression, anxiety, pain, and psychological distress. J Psychiatr Pract 2012;18:233-52.

39. Northoff G, Heinzel A, de Greck M, Bermpohl F, Dobrowolny H, Panksepp J. Self-referential processing in our brain: a meta-analysis of imaging studies on the self. Neuroimage 2006; 31:440-57.

40. McKiernan KA, D’Angelo BR, Kaufman JN, Binder JR. Interrupting the "stream of consciousness": an fMRI investigation. Neuroimage 2006;29:1185-91.

41. Neff K. Self-compassion: an alternative conceptualization of a healthy attitude toward oneself. Self Ident 2003;2:85-101.

42. Hollis-Walker L, Colosimo K. Mindfulness, self-compassion, and happiness in non-meditators: a theoretical and empirical examination. Per Individ Dif 2011;50:222-7.

43. Birnie K, Speca M, Carlson LE. Exploring self-compassion and empathy in the context of mindfulness-based stress reduction (MBSR). Stress Health 2010;26:359-71.

44. Gross CR, Kreitzer MJ, Reilly-Spong M, Wall M, Winbush NY, Patterson R, et al. Mindfulness-based stress reduction versus pharmacotherapy for chronic primary insomnia: a randomized controlled clinical trial. Explore (NY) 2011;7:76-87.

45. Lengacher CA, Reich RR, Paterson CL, Jim HS, Ramesar S, Alinat $\mathrm{CB}$, et al. The effects of mindfulness-based stress reduction on objective and subjective sleep parameters in women with breast cancer: a randomized controlled trial. Psychooncology $2015 ; 24: 424-32$

46. Garland SN, Carlson LE, Stephens AJ, Antle MC, Samuels C, Campbell TS. Mindfulness-based stress reduction compared with cognitive behavioral therapy for the treatment of insomnia comorbid with cancer: a randomized, partially blinded, noninferiority trial. J Clin Oncol 2014;32:449-57.

47. Ong JC, Manber R, Segal Z, Xia Y, Shapiro S, Wyatt JK. A randomized controlled trial of mindfulness meditation for chronic insomnia. Sleep 2014;37:1553-63.

48. Wang YY, Wang F, Zheng W, Zhang L, Ng CH, Ungvari GS, et al. Mindfulness-based interventions for insomnia: a meta-analysis of randomized controlled trials. Behav Sleep Med 2020;18: $1-9$.

49. Rash JA, Kavanagh VA, Garland SN. A meta-analysis of mindfulness-based therapies for insomnia and sleep disturbance: moving towards processes of change. Sleep Med Clin 2019; 14: 209-33.

50. Britton WB, Haynes PL, Fridel KW, Bootzin RR. Polysomnographic and subjective profiles of sleep continuity before and after mindfulness-based cognitive therapy in partially remitted depression. Psychosom Med 2010;72:539-48.

51. Britton WB, Haynes PL, Fridel KW, Bootzin RR. Mindfulness-based cognitive therapy improves polysomnographic and subjective sleep profiles in antidepressant users with sleep complaints. Psychother Psychosom 2012;81:296-304.

52. Ong J, Sholtes D. A mindfulness-based approach to the treatment of insomnia. J Clin Psychol 2010;66:1175-84. 